\title{
Icariin inhibits corticosterone-induced apoptosis in hypothalamic neurons via the PI3-K/Akt signaling pathway
}

\author{
HONGYING ZHANG $^{1 *}$, BAOJUN LIU ${ }^{1 *}$, JINFENG WU $^{1}$, CHANGQING XU $^{1}$, JIANG TAO ${ }^{2}$, \\ XIAOHONG DUAN $^{1}$, YUXUE CAO ${ }^{1}$ and JINGCHENG DONG ${ }^{1}$ \\ ${ }^{1}$ Laboratory of Lung, Inflammation and Cancers, Huashan Hospital, Fudan University, Shanghai 200040; \\ ${ }^{2}$ Department of General Dentistry, Ninth People's Hospital, Shanghai Jiao Tong University School of Medicine, \\ Shanghai Key Laboratory of Stomatology, Shanghai 200011, P.R. China
}

Received March 25, 2012; Accepted August 6, 2012

DOI: $10.3892 / \mathrm{mmr} .2012 .1041$

\begin{abstract}
Excessive corticosterone (CORT) is acknowledged to induce neuronal damage in a number of regions of the brain, particularly the hippocampus, the main area implicated in depression. However, little research has been conducted on alterations to hypothalamic neurons in depression and the cellular and molecular basis for these changes. In the present study, we aimed to determine whether CORT causes apoptosis in primary cultured hypothalamic neurons, and to investigate the protective effects of icariin, an active natural ingredient from the Chinese plant, Epimedium sagittatum Maxim. Our study demonstrates that exposure of hypothalamic neurons to CORT causes a significant loss in viability, a significant decrease in mitochondrial membrane potential, an increase in caspase-3 activity, an elevation in intracellular reactive oxygen species elevation and decreased superoxide dismutase activity. However, pretreatment of cells with icariin prior to CORT exposure was identified to noticeably suppress these CORT-induced events. Furthermore, icariin may prevent CORT-induced cell death via activation of the PI3-K/Akt pathway. In conclusion, icariin is able to prevent CORT-induced hypothalamic cell apoptosis via activation of the PI3-K/Akt pathway.
\end{abstract}

Correspondence to: Professor Jingcheng Dong, Laboratory of Lung, Inflammation and Cancers, Huashan Hospital, Fudan University, Shanghai 200040, P.R. China

E-mail: jcdong2004@hotmail.com

Abbreviations: CORT, corticosterone; PI3-K/Akt, phosphoinositide 3-kinase/protein kinase B; MTT, 3-(4,5)-dimethylthiahiazo(-z-y1)3,5-di-phenytetrazoliumromide; LDH, lactate dehydrogenase; ROS, reactive oxygen species; HPA, hypothalamic-pituitary-adrenal; ICA, icarrin; GR, glucocorticoid receptor; MAPK, mitogen-activated protein kinases; Rho 123, rhodamine 123; MMP, mitochondrial membrane potential; SOD, superoxide dismutase

*Contributed equally

Key words: icariin, hypothalamus, apoptosis, corticosterone, PI3-K/Akt pathway

\section{Introduction}

Depression is one of the most common types of mood disorder and is associated with significant disability and mortality. It is projected to become the second leading cause of burden of disease in $2030(1,2)$. Although a number of theories have been put forward to explain the pathology of depression, including the monoamine hypothesis, the neurotransmitter receptor hypothesis and the neurotrophic factor hypothesis, the precise mechanisms underlying the depression remain unclear (3).

Considerable evidence suggests that dysregulation of the hypothalamic-pituitary-adrenal (HPA) axis is associated with depression $(4,5)$. Hyperactivity of the HPA axis results in increased levels of the glucocorticoid hormone cortisol in depressed patients (6). Studies on the effects of excessive glucocorticoid on the brain in depression have to date focused on neuronal apoptosis. However, the majority of studies have only examined the hippocampal neurons in response to perturbed HPA-axis function, and little research has been conducted on alterations to hypothalamic neurons and the cellular and molecular basis for these changes. Previous studies have revealed that the hypothalami of mice subcutaneously implanted with corticosterone (CORT) pellets exhibited significant changes in the expression of proteins involved in cell death (7). A recent study identified that the antidepressant, sertraline, reduced depression-related behavior and decreased the number of apoptotic cells in the hypothalamus in a rat model of depression following myocardial infarction (8). Therefore, CORT-induced hypothalamic cell damage may correlate with behavioral manifestations of depression. Current antidepressants, which target monoaminergic systems, are widely available on the pharmaceutical market. However, approximately $30 \%$ of patients fail to respond to this therapy (9). Therefore, the search for novel drug targets for the treatment of major depression continues.

Recently, we reported that icariin, a biologically active purified compound from the Chinese herbal plant Epimedium sagittatum Maxim was able to reverse social defeat-induced depression-related behavior in mice, which is potentially mediated through inhibition of CORT secretion and glucocorticoid receptor (GR) downregulation (10). Furthermore, we identified that icariin markedly suppresses 
CORT-induced apoptosis in primary cultured rat hippocampal neurons, presumably through blockade of p38MAPK activation (11) In the present study, we aim to determine whether CORT is responsible for apoptosis in primary cultured hypothalamic neurons, and to investigate the protective effects of icariin by examining the activation of the phosphoinositide 3-kinase/protein kinase B (PI3-K/Akt) signaling pathway.

\section{Materials and methods}

Primary cultures of hypothalamic cells. The experiments were performed on Sprague-Dawley (SD) rats less than $24 \mathrm{~h}$ old purchased from the Shanghai Institute of the Chinese Academy of Science. This study was approved by the Fudan University experimental standards and followed the international guidelines on the ethical treatment of experimental animals. Primary cultures of dissociated hypothalamic cells were prepared according to a previously described method (12) with specific modifications. The hypothalamic tissue was dissected from neonatal SD rats, stripped of the meninges and blood vessels, and minced in Hanks' balanced salt solution (HBSS) without $\mathrm{Ca}^{2+}$ and $\mathrm{Mg}^{2+}$. The tissue was dissociated using $0.125 \%$ trypsin (Beijing Solarbio Science and Technology Co., Ltd., Beijing, China) digestion for $15 \mathrm{~min}$ at $37^{\circ} \mathrm{C}$ and gentle titration through a series of glass pasteur pipettes. The cells were then plated on poly-L-lysine (molecular weight, 30,00070,000; 0.1mg/ml; Sigma, St. Louis, MO, USA) coated-glass coverslips, 96-well plates, or $100 \mathrm{~mm}$ dishes at a density of $1 \times 10^{6}$ cell $/ \mathrm{ml}$ and maintained at $37^{\circ} \mathrm{C}$ in a humidified $5 \% \mathrm{CO}_{2}$ incubator. Neurons were cultured in neurobasal-A medium (Gibco BRL, Grand Island, NY, USA) and supplemented with 2\% B27 supplement (Gibco BRL), $10 \mu \mathrm{l} / \mathrm{ml}$ penicillin-streptomycin, $2 \mathrm{mM}$ glutamine (Solarbio), $5 \mathrm{ng} / \mathrm{ml}$ bovine fibroblast growth factor 2 (FGF2; R\&D Systems, Minneapolis, MN, USA) and $1 \%$ fetal bovine serum (FBS). After a 24-h culture period, cultures grown in serum-free neurobasal medium yielded greater than $80 \%$ neurons, as estimated by immunocytochemical staining with antibodies against neurofilament proteins. The cultured neurons were used for in vitro studies on day 8 (DIV 8).

Drug treatment. CORT (Sigma) was initially dissolved in ethanol as a stock solution and then in culture media (final concentration of ethanol, $0.1 \%$ ). Icariin (purity $>99 \%$ ) was purchased from the Shanghai Winherb Medical S\&T Development Co., Ltd. (Shanghai, China). Eight-day primary hypothalamic neurons were washed twice with $\mathrm{Mg}^{2+}$-free, HEPES-buffered saline (HBS; $146 \mathrm{mM} \mathrm{NaCl}, 10 \mathrm{mM}$ HEPES, $2 \mathrm{mM} \mathrm{CaCl}_{2}, 5 \mathrm{mM} \mathrm{KCl}, 10 \mathrm{mM}$ D-glucose, $\mathrm{pH}$ 7.4) and pretreated with different concentrations of icariin with or without $50 \mu \mathrm{M}$ LY294002 (Sigma) for $2 \mathrm{~h}$ at $37^{\circ} \mathrm{C}$, and then exposed to CORT for $24 \mathrm{~h}$.

Assessement of cell viability and death. After exposure to various concentrations of CORT, icariin and LY294002, cell viability was determined using the 3-(4,5-dimethylthiazol2-yl)-2,5-diphenyl (MTT) assay system (Beyotime Institute of Biotechnology, Shanghai, China) (11). Cell cytotoxicity following various treatments was evaluated by lactate dehydrogenase $(\mathrm{LDH})$ release. This was acheived using a Quantitative
Detection kit according to the manufacturer's instructions (GenMed Scientifics Inc., Arlington, MA, USA) (11).

Cell morphology evaluation. To evaluate the morphological changes in primary cultures of hypothalamic cells, cultures were observed under a Leica DFIL inverted microscope with a phase-contrast optic lens. Images were captured using Leica QWin plus 3 Image Processing Software (Media Cybernetics, Silver Spring, MD, USA) through a Leica DFC300 FX camera device. We analyzed neurite preference from five images per condition.

Measurement of caspase-3 activity. Caspase-3 activity in primary cultures of hypothalamic lysate was determined using the Chemicon caspase colorimetric activity assay kit according to the manufacturer's instructions (11). In brief, treated cells were resuspended in $50 \mu \mathrm{l}$ of cell lysis buffer and incubated on ice for $30 \mathrm{~min}$. After centrifugation for $5 \mathrm{~min}$ at $10,000 \mathrm{x} \mathrm{g}$, the supernatant was transferred to a fresh tube followed by the addition of reaction buffer and caspase- 3 substrate for $4 \mathrm{~h}$ at $37^{\circ} \mathrm{C}$ in the dark. The free chromophore $p$-nitroaniline ( $p \mathrm{NA}$ ) was quantified using a microtiter plate reader at $405 \mathrm{~nm}$. The fold increase in caspase-3 activity was determined by comparing the absorbance from each sample with untreated neurons.

Determination of mitochondrial membrane potential (MMP). Following treatment, hypothalamic cell cultures grown on 96-well plates were loaded with Rhodamine 123 (Rho 123) $(10 \mu \mathrm{M})$ (Beyotime Institute of Biotechnology, Shanghai, China) at $37^{\circ} \mathrm{C}$ in the dark for $15 \mathrm{~min}$ and then washed 3 times with phosphate-buffered saline (PBS). The cell fluorescence intensity of Rho 123 was quantified using a fluorescence microplate reader (TECAN Infinite 200 microplate reader; Tecan Trading AG, Männedorf, Switzerland) with excitation at $485 \mathrm{~nm}$ and emission at $530 \mathrm{~nm}$. The background fluorescence signal of Rho 123 was determined without cells and subtracted from those obtained in hypothalamic neurons.

Analysis of intracellular reactive oxygen species (ROS). Intracellular ROS levels were analyzed by H2DCF-DA assay according to the method previously described (13). Cells $\left(1 \times 10^{6}\right.$ cells/well) were rinsed with Kreb's ringer solution and $10 \mathrm{mM}$ H2DCF-DA was added. After $15 \mathrm{~min}$ incubation at $37^{\circ} \mathrm{C}$, cells were washed with PBS, harvested and pelleted by centrifugation and then resuspended in $0.5 \mathrm{ml} \mathrm{PBS}$. Fluorescence intensity was then monitored using a fluorescence microplate reader. The data was analyzed and expressed as a percentage of the control. DCF labeled cells were observed under fluorescence microscopy.

Measurement of antioxidant enzyme activities. Following treatment, the cultures were washed twice with PBS, then placed into ice-cold PBS (0.1 M, containing 0.05 mM EDTA) and homogenized. The homogenate was then centrifuged at $4^{\circ} \mathrm{C}$ at $10,000 \times \mathrm{g}$ for $30 \mathrm{~min}$, after which the protein concentration was determined using the Bradford method, using bovine serum albumin (BSA) as a reference standard. Measurement of superoxide dismutase (SOD) activity was performed according to the reagent kit manufacturer's instructions. 
Western blot analysis. Following treatment, cells in each of the 6-well plates were rinsed twice with cold PBS, followed by the addition of cell lysis buffer containing $150 \mathrm{mM} \mathrm{NaCl}$, $50 \mathrm{mM}$ Tris-HCl, 5 mM EDTA, 1\% Nonidet P-40, $0.5 \%$ deoxycholate, $1 \%$ SDS with proteinase inhibitor cocktail (Sigma) on ice for $15 \mathrm{~min}$, and then centrifuged for $20 \mathrm{~min}$ at $12,000 \mathrm{x}$. The supernatant was collected and the protein concentration was measured using the Bradford method. Fifty milligrams of total protein was dissolved in sample buffer and heated for 5 min prior to loading onto polyacrylamide gels. Proteins were then transferred to poly(vinylidine difluoride) filter membranes, and blocked with $5 \%$ non-fat dry milk in Tris-buffered saline $/ 0.05 \%$ Tween-20. The membrane was incubated with a monoclonal antibody against phospho-Akt (Ser473), Akt (Santa Cruz Biotechnology Inc., Santa Cruz, CA, USA), followed by incubation with horseradish peroxidase-conjugated (HRP) secondary antibodies and visualized using an enhanced chemiluminescence kit.

Statistical analysis. All data are expressed as the mean \pm SD. Differences between groups without particular comments were generally examined for statistical significance using one-way ANOVA analysis with a post-hoc Dunnett's test. $\mathrm{P} \leq 0.05$ indicated a statistically significant difference.

\section{Results}

Icariin protects hypothalamic neurons against CORT-induced cytotoxicity. Cultured hypothalamic neurons were exposed to an increasing concentration of CORT $(0.01-5 \mu \mathrm{M})$ for $24 \mathrm{~h}$ and cell viability was assessed using an MTT reduction assay. CORT induced cell death in a concentration-dependent manner (Fig. 1A). Exposure to CORT $(1 \mu \mathrm{M})$ for $24 \mathrm{~h}$ was used for inducing excitotoxic neuronal injury in the subsequent experiments.

To investigate the neuroprotective effects of icariin on CORT-induced neuronal damage, hypothalamic neurons were pretreated with icariin at $0.1 \mu \mathrm{M}$ and $1 \mu \mathrm{M}$ for $2 \mathrm{~h}$, followed by challenge with CORT $(1 \mu \mathrm{M})$ for $24 \mathrm{~h}$. Icariin pretreatment at $0.1 \mu \mathrm{M}$ and $1 \mu \mathrm{M}$ enhanced cell viability compared with the CORT-treated control (Fig. 1B). The protective effect of icariin on CORT-induced cytotoxicity was also analyzed using the LDH test. Pretreatment with $0.1 \mu \mathrm{M}$ and $1 \mu \mathrm{M}$ icariin caused a marked decrease in LDH release (Fig. 1C).

Icariin suppresses hypothalamic neuronal apoptosis induced by CORT. Morphological analysis at the subcellular level remains the most conclusive method for distinguishing apoptosis from necrosis. The protective effect of icariin on CORT-induced cytotoxicity was also analyzed by microscopic examination. Cultured hypothalamic neurons were pretreated with or without $1 \mu \mathrm{M}$ icariin for $2 \mathrm{~h}$, followed by challenge with CORT $(1 \mu \mathrm{M})$ for $24 \mathrm{~h}$. Cells treated with vehicle were healthy with networks of neurites and vacuole-free cell bodies. The synapse connections between neurons were clearly observed. Neurite fragmentation, shrinkage of cell bodies and evident cell loss were observed when the neuronal culture was exposed to $1 \mu \mathrm{M}$ CORT for $24 \mathrm{~h}$. Pretreatment of icariin with $1 \mu \mathrm{M}$ was able to protect cortical neurons against CORT toxicity as demonstrated by the fine morphology (Fig. 2A).
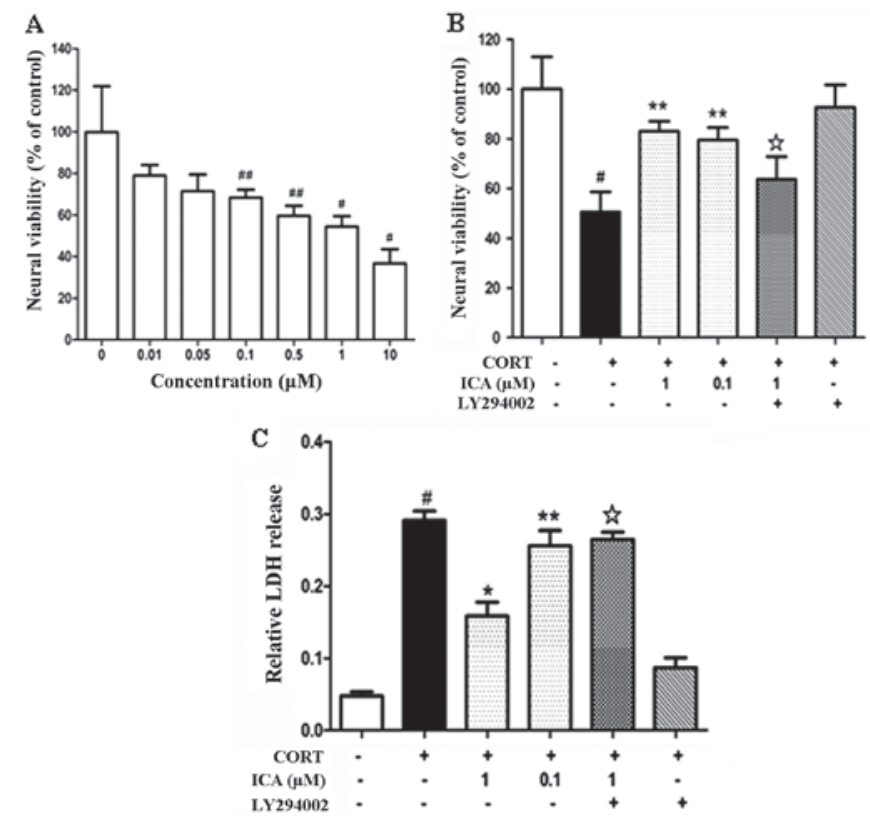

Figure 1. Icariin protected primary cultured neuronal cells from CORT-induced neurotoxicity. (A) CORT-induced reduction viability of primary cultured neuronal cells in a dose-dependent manner. Eight-day-old primary hypothalamic neurons were treated with icariin for $2 \mathrm{~h}$ prior to a $24-\mathrm{h}$ exposure to $1 \mu \mathrm{M}$ CORT. Following treatment, (B) cell viability and (C) LDH release were measured. Data represents the mean $\pm \mathrm{SD}$ of three independent experiments, each was performed in triplicate. Statistical significance using a t-test was set as ${ }^{\# \#} \mathrm{P}<0.05,{ }^{\#} \mathrm{P}<0.01$ vs. vehicle control, ${ }^{* *} \mathrm{P}<0.05,{ }^{*} \mathrm{P}<0.01$ vs. model, ${ }^{4} \mathrm{P}<0.01$ vs. icariin group. CORT, corticosterone; ICA, icariin; $\mathrm{LDH}$, lactate dehydrogenase.

Biochemical markers for caspase-3 activation following treatment with CORT were consistent with apoptotic cell death. Following 24-h CORT treatment, caspase-3 activity increased in the hypothalamic neurons. In the cultured hypothalamic neurons exposed to $1 \mu \mathrm{M}$ CORT plus $1 \mu \mathrm{M}$ icariin, caspase- 3 activity was similar to that the of the control (Fig. 2B)

Mitochondria are recognised to be involved in apoptosis. Permeability changes can lead to caspase-dependent cytotoxicity and downstream apoptotic signaling, while a loss of mitochondrial transmembrane potential, denoted as mitochondrial dysfunction, leads to cytochrome c release from the mitochondria and triggers other apoptotic factors. In the present study, we evaluated mitochondrial transmembrane potential using Rho 123 as fluorescent dye. Dye distribution was examined under inverted microscope (Leica DFIL). In the control cultures, Rho 123 was markedly aggregated in hypothalamic neurons, reflecting the baseline for healthy mitochondria in cells. CORT-treated neurons demonstrated a decrease in Rho 123 of $37 \%$. Pretreatment of $1 \mu \mathrm{M}$, but not $0.1 \mu \mathrm{M}$ icariin, significantly inhibited these changes (Fig. 2C and D).

Icariin inhibits intracellular accumulation of ROS and prevents loss of antioxidant enzyme activities in CORT-treated cells. To determine whether icariin attenuates cell death by blocking ROS generation, we detected the intracellular level of ROS using H2DCF-DA fluorescent dye. Treatment of hypothalamic neurons with $1 \mu \mathrm{M}$ CORT for $24 \mathrm{~h}$ resulted in a significantly increased DCF signal compared with the control 
A

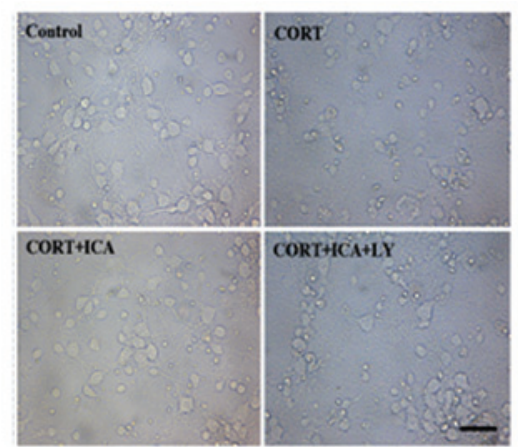

C

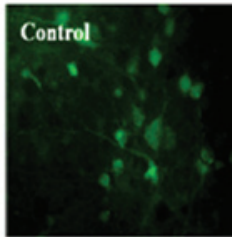

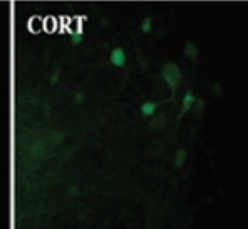

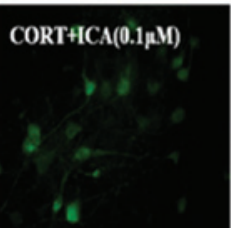

CORT+ICA+LY

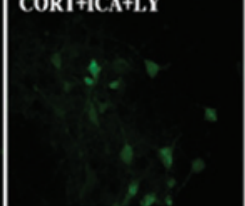

B

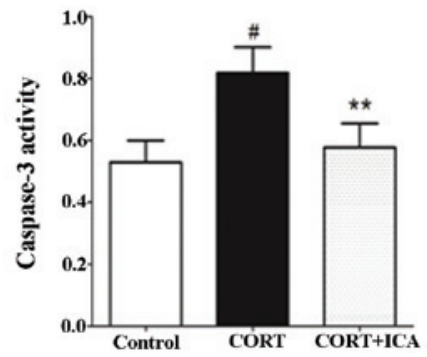

$\mathrm{D}$

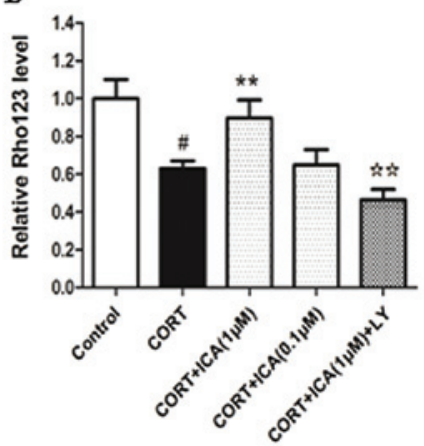

Figure 2. Icariin inhibited CORT-induced apoptosis in cultured hypothalamic neurons. (A) Morphological characteristics of cultured cortical neurons were visualized under microscopic analysis. Scale bar $=50 \mu \mathrm{M}$. (B) Activation of caspase-3. (C) Mitochondrial function was determined by Rho 123 fluorescence intensity. Scale bar $=1 \mathrm{~mm}$ for all images. (D) Rho 123 fluorescence intensity was quantitatively analyzed. Data are expressed as fold over the control group in five to six coverslips from separate cultures. Statistical significance using a t-test was set as ${ }^{\# \#} \mathrm{P}<0.05,{ }^{\# P} \mathrm{P}<0.01$ vs. vehicle control, ${ }^{* *} \mathrm{P}<0.05$, ${ }^{*} \mathrm{P}<0.01$ vs. model, 蛴 $\mathrm{P}<0.01$ vs. icariin group. CORT, corticosterone; ICA, icariin; LY, LY294002.

A
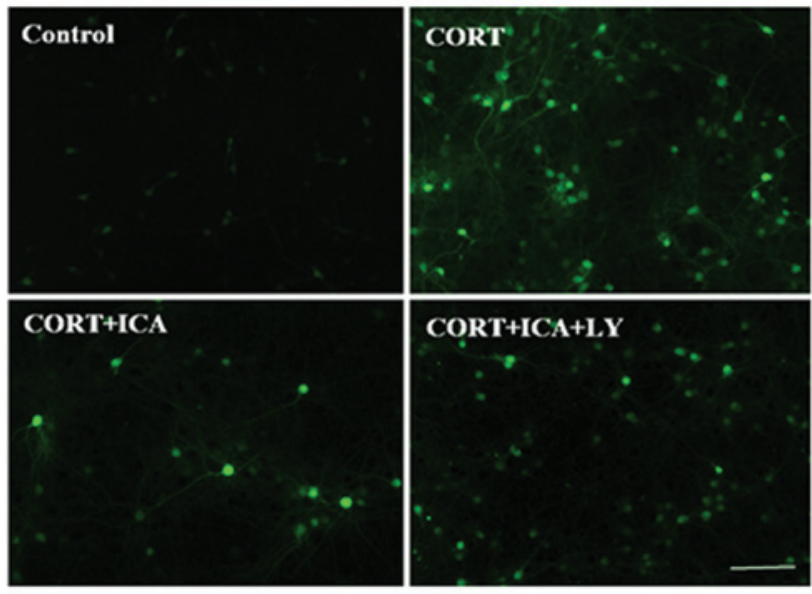

B

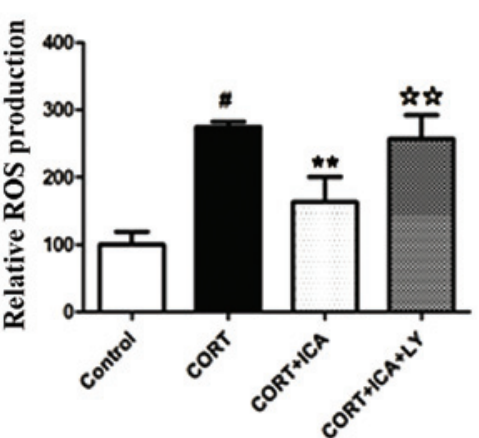

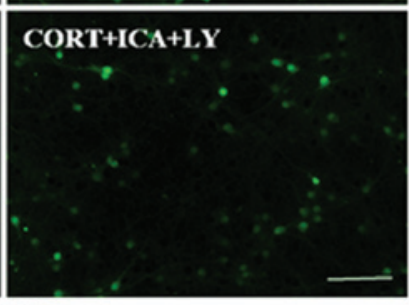

C

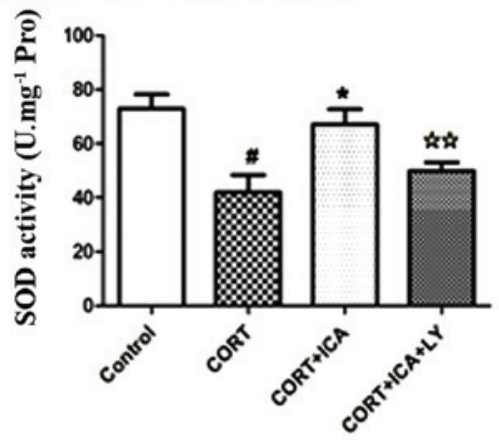

Figure 3. Icariin inhibited CORT-induced intracellular accumulation of ROS and rescued loss of SOD. (A) Intracellular ROS were imaged using the H2DCF-DA fluorescent dye. (B) Quantitative analysis of ROS in cortical neurons was determined by fluorescence microplate reader. (C) Measurement of the SOD. Statistical significance using a t-test was set as ${ }^{\# \#} \mathrm{P}<0.05,{ }^{~} \mathrm{P}<0.01$ vs. vehicle control, ${ }^{* *} \mathrm{P}<0.05,{ }^{*} \mathrm{P}<0.01$ vs. model, ${ }^{\text {站 }} \mathrm{P}<0.01$ vs. icariin group. CORT, corticosterone; ICA, icariin; ROS, reactive oxygen species; SOD, superoxide dismutase; LY, LY294002. 


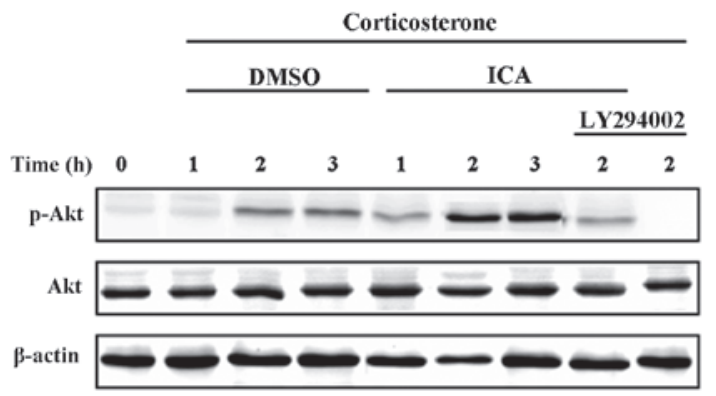

Figure 4. Effects of corticosterone and ICA on the levels of phosphorylated Akt and total Akt in cultured hypothalamic neurons. The intensity of the bands for the phosphorylation of Akt and total Akt was analyzed by western blot analysis. The experiments were repeated three times independently, one representative result is shown. ICA, icariin.

group, but this was significantly reduced by icariin pretreatment (Fig. 3A and B).

The level of SOD provided further evidence of the protective effects of icariin. Incubation with $1 \mu \mathrm{M}$ CORT for $24 \mathrm{~h}$ resulted in a significantly decreased activity of SOD (42.5\%) in the hypothalamic neurons compared to the controls. However, pretreatment with $1 \mu \mathrm{M}$ icariin resulted in a significant increase in the activity of SOD (37.5\%) when compared to cells treated with CORT. Together, these results suggest that pretreatment with icariin prevents ROS generation and attenuates changes in SOD activity induced by treatment with CORT (Fig. 3C).

Phosphorylation of Akt in CORT-treated neurons following exposure to icariin. Activation of the PI3-K/Akt signal pathway by CORT and icariin in cultured hypothalamic neurons was determined by measuring phospho-Akt levels. Neurons were pretreated with icariin or control media for $30 \mathrm{~min}$ followed by stimulation with CORT for 1, 2 and $3 \mathrm{~h}$. Western blot analysis revealed a significant increase in phospho-Akt at $2 \mathrm{~h}$ following treatment with CORT in cultured hypothalamic neurons, similar levels were also recorded in the cells treated for $3 \mathrm{~h}$. In the icariin-treated groups, Akt phosphorylation was increased compared with the CORT-treated control group. As expected, the cells treated with LY294002 demonstrated a significant decrease in the amount of phosphorylated Akt at $2 \mathrm{~h}$ (Fig. 4).

\section{Discussion}

The present study demonstrates that icariin prevents CORT-induced apoptosis in primary cultured hypothalamic neurons. Exposure of hypothalamic neurons to CORT resulted in a significant loss of viability and apoptosis of the cells. In parallel, CORT significantly increased the intracellular ROS elevation and decreased SOD activity. However, pretreatment of the cells with icariin prior to CORT exposure noticeably suppressed these CORT-induced cellular events. Furthermore, icariin is able to prevent CORT-induced hypothalamic cell death via activation of the PI3-K/Akt pathway.

Patients with major depression and other neurological afflictions frequently display hyperactivity of the HPA axis (14). Patients with major depression are known to have a higher level of cortisol than patients without major depression (15). In rodents, studies have revealed that repeated CORT injections induce behavioral and neurochemical aspects of depression (16). For example, chronic CORT injections increased the immobility time on the forced swimming test (17). Several studies have demonstrated that glucocorticoids induce atrophy of dendritic processes, inhibition of neurogenesis and cause overt loss of neurons by necrosis or apoptosis of the hippocampus (18-20). Our recent study also revealed that CORT may induce hippocampal neuronal damage (11). In the present study, we observed morphological changes, including displayed neurite fragmentation, shrinkage of cell bodies and evident cell loss in hypothalamic neurons following CORT treatment. Furthermore, our results demonstrated that exposure to CORT significantly decreased MMP and increased caspase-3 activity. These results are consistent with previous studies that the hypothalami of mice implanted with CORT pellets exhibited significant changes in proteins related to cell death pathways (7).

Several studies have demonstrated that ROS may lead to neuronal apoptosis in neurodegenerative disorders (21). ROS is a natural by-product of the normal metabolism of oxygen in the mitochondria, and accumulation of ROS may be related to mitochondrial dysfunction and the induction of apoptosis (22). However, the antioxidant enzymes of SOD play protective roles in all aerobic organisms by scavenging the generation of free radical molecules. An imbalance between the generation of free radicals and antioxidants may be involved in the pathogenesis of most neurodegenerative diseases. In agreement with previously reported results, our study demonstrated that CORT induces ROS generation and suppresses SOD activity. The results suggest ROS are involved in CORT-induced cytotoxicity.

The involvement of the PI3-K/Akt signal pathway in neuronal survival involves different mechanisms. To identify the intracellular signaling pathways that mediate the neuroprotective actions of icariin, changes in the phosphorylation of key signaling proteins were analyzed by immunoblots with phospho-specific antibodies. In the present study, neurons were pretreated with icariin or control media for $30 \mathrm{~min}$ followed by stimulation with CORT for 1,2 and $3 \mathrm{~h}$. Western blot analysis revealed an increase in phospho-Akt at $2 \mathrm{~h}$ following treatment with CORT in cultured hypothalamic neurons and these levels of phospho-Akt were sustained for at least $3 \mathrm{~h}$. This is consistent with previous studies that revealed CORT increases the phosphorylation of PI3-K/Akt in neurons grown in neurobasal medium supplemented with B27 and $500 \mu \mathrm{m}$ L-glutamine (NBM+) (23). In the icariin-treated groups, Akt phosphorylation was increased compared with the CORTtreated control group. As expected, the cells treated with LY294002 demonstrated a significant decrease in the amount of phosphorylated Akt at $2 \mathrm{~h}$. In addition, pretreatment with LY294002 significantly blocked the neuroprotective effects of icariin against CORT-induced apoptosis. These results suggest that icariin is able to prevent neuronal cell death induced by CORT in hypothalamic neurons by modulating the activity of the PI3-K/Akt pathway.

In conclusion, icariin was identified to be able to prevent CORT-induced hypothalamic cell death via activation of the PI3-K/Akt pathway. This study not only demonstrates the 
potential pharmacological uses of icariin, but also reveals the key neuroprotective role of the PI3-K/Akt pathway.

\section{Acknowledgements}

This study was supported by a grant from the National Basic Science Program of China (No. 2009CB523000) and the National Natural Science Foundation of China (No. 81102562).

\section{References}

1. Mathers CD and Loncar D: Projections of global mortality and burden of disease from 2002 to 2030. PLoS Med 3: e442, 2006.

2. Ustun TB, Ayuso-Mateos JL, Chatterji S, Mathers C and Murray CJ: Global burden of depressive disorders in the year 2000. Br J Psychiatry 184: 386-392, 2004.

3. Chopra K, Kumar B and Kuhad A: Pathobiological targets of depression. Expert Opin Ther Targets 15: 379-400, 2011.

4. Pariante CM and Lightman SL: The HPA axis in major depression: classical theories and new developments. Trends Neurosci 31: 464-468, 2008.

5. Snyder JS, Soumier A, Brewer M, Pickel J and Cameron HA: Adult hippocampal neurogenesis buffers stress responses and depressive behaviour. Nature 476: 458-461, 2011.

6. Pariante CM: Risk factors for development of depression and psychosis. Glucocorticoid receptors and pituitary implications for treatment with antidepressant and glucocorticoids. Ann NY Acad Sci 1179: 144-152, 2009.

7. Skynner HA, Amos DP and Murray F, et al: Proteomic analysis identifies alterations in cellular morphology and cell death pathways in mouse brain after chronic corticosterone treatment. Brain Res 1102: 12-26, 2006

8. Wann BP, Bah TM and Kaloustian S, et al: Behavioural signs of depression and apoptosis in the limbic system following myocardial infarction: effects of sertraline. J Psychopharmacol 23: 451-459, 2009

9. Kulkarni SK and Dhir A: Current investigational drugs for major depression. Expert Opin Investig Drugs 18: 767-788, 2009.

10. Wu J, Du J and Xu C, et al: Icariin attenuates social defeatinduced down-regulation of glucocorticoid receptor in mice. Pharmacol Biochem Behav 98: 273-278, 2011.

11. Liu B, Zhang $\mathrm{H}$ and $\mathrm{Xu} \mathrm{C}$, et al: Neuroprotective effects of icariin on corticosterone-induced apoptosis in primary cultured rat hippocampal neurons. Brain Res 1375: 59-67, 2011.
12. Yokosuka M, Ohtani-Kaneko R, Yamashita K, Muraoka D, Kuroda Y and Watanabe C: Estrogen and environmental estrogenic chemicals exert developmental effects on rat hypothalamic neurons and glias. Toxicol In Vitro 22: 1-9, 2008.

13. Wang $\mathrm{H}, \mathrm{Xu} \mathrm{Y}$ and Yan J, et al: Acteoside protects human neuroblastoma SH-SY5Y cells against beta-amyloid-induced cell injury. Brain Res 1283: 139-147, 2009.

14. Kunugi $\mathrm{H}$, Hori $\mathrm{H}$, Adachi $\mathrm{N}$ and Numakawa T: Interface between hypothalamic-pituitary-adrenal axis and brain-derived neurotrophic factor in depression. Psychiatry Clin Neurosci 64: 447-459, 2010.

15. Keller J, Flores B and Gomez RG, et al: Cortisol circadian rhythm alterations in psychotic major depression. Biol Psychiatry 60: 275-281, 2006.

16. Iijima M, Ito A, Kurosu S and Chaki S: Pharmacological characterization of repeated corticosterone injection-induced depression model in rats. Brain Res 1359: 75-80, 2010.

17. Ago Y, Arikawa S and Yata M, et al: Antidepressant-like effects of the glucocorticoid receptor antagonist RU-43044 are associated with changes in prefrontal dopamine in mouse models of depression. Neuropharmacology 55: 1355-1363, 2008.

18. Gerritsen L, Comijs HC, van der Graaf Y, Knoops AJ, Penninx BW and Geerlings MI: Depression, hypothalamic pituitary adrenal axis, and hippocampal and entorhinal cortex volumes - The SMART Medea Study. Biol Psychiatry 70: 373-380, 2011.

19. de Quervain DJ, Aerni A, Schelling G and Roozendaal B: Glucocorticoids and the regulation of memory in health and disease. Front Neuroendocrinol 30: 358-370, 2009.

20. Xu Y, Zhang C and Wang R, et al: Corticosterone induced morphological changes of hippocampal and amygdaloid cell lines are dependent on 5-HT7 receptor related signal pathway. Neuroscience 182: 71-81, 2011.

21. Fatokun AA, Stone TW and Smith RA: Oxidative stress in neurodegeneration and available means of protection. Front Biosci 13: 3288-3311, 2008.

22. Huang SH, Lin CM and Chiang BH: Protective effects of Angelica sinensis extract on amyloid beta-peptide-induced neurotoxicity. Phytomedicine 15: 710-721, 2008.

23. Zhu ZH, Yang R, Fu X, Wang YQ and Wu GC: Astrocyteconditioned medium protecting hippocampal neurons in primary cultures against corticosterone-induced damages via PI3-K/Akt signal pathway. Brain Res 1114: 1-10, 2006. 